\title{
A method to estimate the effect of line blanketing in NLTE radiative transfer calculations
}

\author{
I. Busá ${ }^{1}$, V. Andretta ${ }^{1}$, M. T. Gomez ${ }^{1}$, and L. Terranegra ${ }^{1}$ \\ Osservatorio Astronomico di Capodimonte, Via Moiariello 16, 80131 Napoli, Italy \\ Received 5 February 2001 / Accepted 2 May 2001
}

\begin{abstract}
We present a method to estimate the contribution of line opacity to the total opacity as a function of wavelength. The estimated line-opacity function can then be used to simulate line-blanketing in NLTE radiative transfer calculations. Given a reference flux distribution (either observed or theoretical), our method allows to obtain a good estimate of the spectrum without the need for considering in detail all the millions of lines contributing to line blanketing. We applied the method to the spectra computed from a sample of photospheric models with effective temperatures $T_{\text {eff }}=4200,5200$ and $6200 \mathrm{~K}, \log g=4.0,4.5,5.0$ and $[\mathrm{A} / \mathrm{H}]=0.0,-1.0,-2.0$, taken from the NextGen database (Allard \& Hauschildt 1995). The computed flux distributions agree quite well with the corresponding LTE line-blanketed NextGen fluxes when we introduce the estimated line-opacity contribution as a multiplicative factor of the continuum opacity in the radiative transfer calculations. In particular we discuss the importance of a correct estimate of the continuum flux, mainly in the UV, in the NLTE formation of the Ca II H \& K, the Ca II InfraRed Triplet (IRT: $\lambda=8498,8542,8662$ ), Na I D, Li I and K I resonance lines.
\end{abstract}

Key words. stars: atmospheres - radiative transfer - line: formation - ultraviolet: stars

\section{Introduction}

It has been well known since the first works on non-LTE (NLTE) radiative transfer (e.g., Thomas 1957), that the source function of a line can be strongly coupled to radiation fields even at very different wavelengths, via radiative rates determining the statistical equilibrium of the species producing the line. For the correct evaluation of the relevant radiation fields, however, it is often necessary to take into account line blanketing (Andretta 1997; Short \& Doyle 1997; Mauas et al. 1997; Falchi \& Mauas 1998).

The problem of constructing line-blanketed atmospheres is much easier assuming LTE, because the total line-opacity is then a function of only two variables, the temperature and the electron density. Nevertheless, having to deal with millions of lines, it is necessary to adopt some statistical method such as the opacity distribution function (ODF) or the opacity-sampling (OS) which represents a resampled total-opacity in a given frequency interval. Widely used grids of LTE line-blanketed model atmospheres have been computed by Kurucz (1992) and, more recently, by Allard \& Hauschildt (1995) who computed a grid of cool stars blanketed models (NextGen database) using the PHOENIX code. Both sets of LTE model atmospheres provide an adequate description of the observed continuum in the visible and near-UV (Prieto \& Lambert 2000; Allard \& Hauschildt 1995), indicating that NLTE effects, which dominate in line formation, are not very important in determining the radiation emerging at those wavelengths.

Attempts to include line blanketing in NLTE radiative transfer calculations have been done by many authors. Andretta et al. (1997) examined the formation of Na I lines in M dwarfs with the NLTE code MULTI (Carlsson 1986) including line opacities from PHOENIX. Falchi \& Mauas (1998) obtained NLTE line-blanketed synthetic spectra adopting the Kurucz opacity-package. Hubeny \& Lanz (1995) developed a numerical method for computing NLTE line-blanketed models using the idea of "superlevels" and "superlines", introduced originally by Anderson (1989), to construct the ODF.

More recently, Hauschild et al. (1999) showed how high-resolution profiles of some lines in the spectra of giant stars are sensitive to the effect of both spherical geometry and of line blanketing, via NLTE effects. Schweitzer et al. (2000) applied superlevel formalism to the study in M dwarfs of NLTE line-blanketing due to molecules, while Barman et al. (2000) adopted a direct OS method, employing modern numerical techniques (such as vectorized and parallelised block algorithms), to the construction of NLTE-line blanketed model atmospheres for white dwarfs in cataclysmic variables, where a large number of metals must be included in the calculations and allowed to depart from their LTE populations.

Send offprint requests to: I. Busá, e-mail: ebu@na.astro.it 
Clearly, the the proper treatment of line-blanketing is a key factor in the construction of realistic model atmospheres. Nevertheless, in some applications of more limited scope, line-blanketing enters in NLTE calculations mainly through the photoexcitation/photoionization rates. Thus, it may be interesting to find a simpler, more agile approach that allows a reasonably approximate treatment of the problem. This is especially true with codes such as MULTI, which are not designed to deal with complicated opacity sources, but that allow a detailed NLTE analysis of spectroscopic diagnostics of astrophysical interest. The subject of this research note is precisely such an approximate treatment of line-blanketing.

\subsection{The method}

This work is part of a study in progress on NLTE modelling of the Ca II IRT, Na I and K I lines in main-sequence, solar-type stars. Therefore, we selected from the NextGen database a grid of photospheric models with effective temperatures $T_{\text {eff }}=4200,5200$ and $6200 \mathrm{~K}$, gravity $\log g=4.0,4.5,5.0$, and metallicity relative to the Sun $[\mathrm{A} / \mathrm{H}]=0.0,-1.0,-2.0$. In order to make our NLTE analysis of alkali and alkali-like atoms more complete, we also added Li I. We included hydrogen in our calculations because we made a few tests adding the hydrostatic equilibrium equation (HSE) to recompute the electron density from the original temperature distribution.

The coupled equations of radiative transfer and statistical equilibrium were solved for the $\mathrm{H}, \mathrm{Ca}, \mathrm{Na}, \mathrm{K}$ and Li Atomic models using the version 2.2 of the code MULTI. The opacity package included in the code takes into account free-free opacity, Rayleigh scattering, and boundfree transitions from hydrogen and metals. As far as line-opacity (line-blanketing) is concerned, however, this version of the code allows the inclusion of only up to 150 lines to be considered in detail.

The atomic model for hydrogen consists of 16 levels, 83 lines and 9 bound-free $(b-f)$ transitions in detail. The Ca Model consists of 14 levels, 9 lines and $13 b-f$ transitions, the Na Atom of 12 levels, 28 lines and $11 b-$ $f$ transitions, the KAtom of 13 levels, 19 lines and 12 $b-f$ transitions and the Li Model consists of 19 levels, 52 lines and $18 b-f$ transitions. Calcium collisional data are from Drake (1991) and calcium damping parameters are from Smith \& Drake (1990). Sodium and potassium models are the same as those of Tripicchio et al. (1999). Lithium atomic data are from Carlsson et al. (1994). The adopted abundances are $\log \epsilon_{\odot}(\mathrm{Ca})=6.34$ (Chmielewski $2000), \log \epsilon_{\odot}(\mathrm{Na})=6.31, \log \epsilon_{\odot}(\mathrm{K})=5.13$ (Grevesse \& Anders 1991). We adopted two values for $\log \epsilon(\mathrm{Li}): 1.0$ and 3.0, not scaled according to $[\mathrm{A} / \mathrm{H}]$.

Given the temperature and electron densities from each of the 27 photospheric models, we solved the radiative transfer equations for the $\mathrm{H}, \mathrm{Ca}, \mathrm{Na}, \mathrm{K}$ And $\mathrm{Li}$, and compared the calculated continuum flux distributions with the correspondent line-blanketed NextGen spectra.

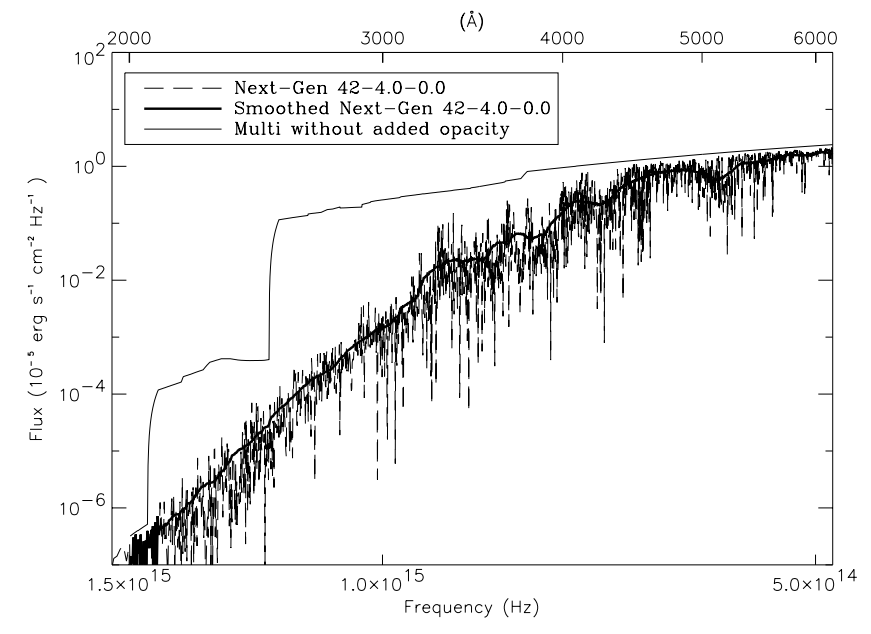

Fig. 1. Comparison between the NextGen (line-blanketed) spectrum (long-dashed line) and the NLTE flux distribution obtained taking into account continuum opacities only (solid thin line), for a photospheric model of $T_{\text {eff }}=4200 \mathrm{~K}, \log g=$ 4.0 and $[\mathrm{A} / \mathrm{H}]=0.0$. The solid thick line shows the running average of the NextGen spectrum over 101 points $(\approx 200 \AA)$.

Figure 1 shows the calculated flux distribution obtained by NLTE radiative transfer calculations (solid thin line) overlapped with the line-blanketed NextGen spectrum for an atmospheric model of $T_{\text {eff }}=4200 \mathrm{~K}, \log g=$ 4.0 and $[\mathrm{A} / \mathrm{H}]=0.0$. The lack of opacity, in the NLTE calculation, produces an overestimate of the UV continuum up to six orders of magnitude with respect to the line-blanketed LTE distribution. This is the worst case in our grid of models: in hotter or less metal-rich models the discrepancy is smaller.

Because we are dealing with the same temperature, pressure and electron density distributions, and neglecting NLTE effects in the formation of the continua, we can assume that the difference in the flux distributions shown in Fig. 1 is only due the different opacity distribution adopted. Moreover, since the opacity package used in MULTI provides a fairly complete description of continuum opacity sources, we can assume that the main difference is due to the lack of line blanketing in MULTI.

We attempted to correct for this missing opacity in MULTI taking the following simple model as a guide.

We indicate with $F_{\mathrm{NLB}}$ (Non Line-Blanketed) the flux distribution calculated by the MULTI code taking into account the continuum-opacity only, and with $F_{\mathrm{LB}}$ (LineBlanketed) the NextGen flux distribution. Approximating the stellar atmosphere as an opaque slab of thickness $\Delta h$ and constant opacity $\kappa$, we can express the emerging flux as $F \propto \exp (-\kappa \Delta h)$, both in the line-blanketed case (LB), and in the case with continuum opacity sources only (NLB).

If we suppose that $\kappa_{\mathrm{LB}}$ can be written in terms of $\kappa_{\mathrm{NLB}}$ and of a wavelength dependent correction $f_{\text {corr }}(\lambda)$ as:

$\kappa_{\mathrm{LB}}=\kappa_{\mathrm{NLB}}\left[1+f_{\text {corr }}(\lambda)\right]$ 


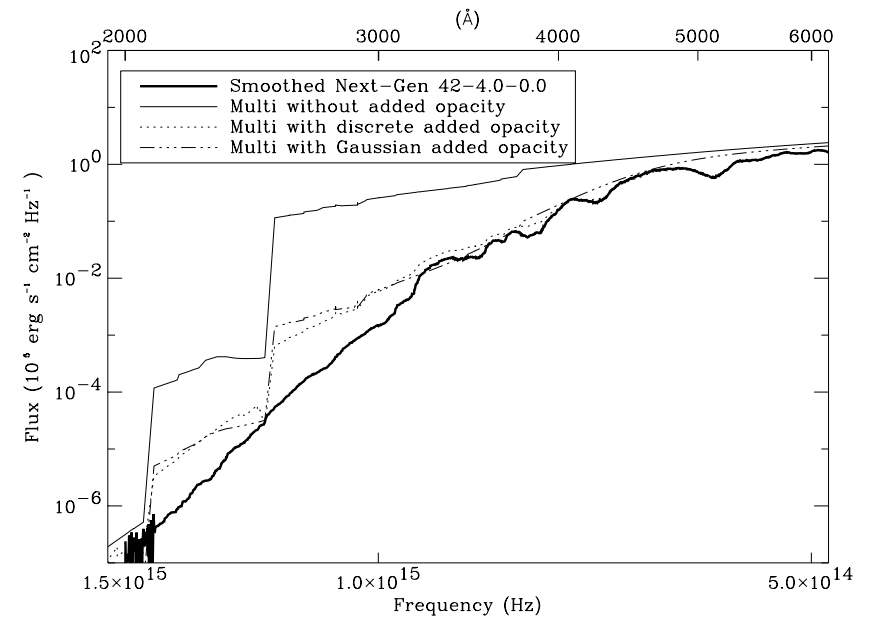

Fig. 2. Comparison between the smoothed NextGen flux (solid thick line), the flux obtained using the original opacity (solid thin line) and the fluxes obtained using the modified $\kappa_{\text {total }}$ opacity function (dotted line) and the Gaussian fit to it (dotdashed line), for the model of Fig. 1.

we find that, in those bands where $\kappa_{\mathrm{NLB}}$ (essentially due to continuum processes) does not vary strongly with wavelength, the correction term $f_{\text {corr }}(\lambda)$ is given by:

$f_{\text {corr }}(\lambda)=$ const $\times \ln \left(F_{\mathrm{NLB}} / F_{\mathrm{LB}}\right)$.

On this basis, we corrected the opacity computed by MULTI as follows:

$\kappa_{\text {total }}=\kappa_{\mathrm{NLB}}\left[1+\right.$ const $\left.\times \ln \left(F_{\mathrm{NLB}} / F_{\mathrm{LB}}\right)\right]$.

We applied the above correction to the wavelength range $1800-30000 \AA$, adopting const $=2 / 3$, which is found to give the best agreement.

The continuum flux distribution calculated, for the model of Fig. 1, using the total opacity of Eq. (3) is shown in Fig. 2 (dotted line). It is clear that the new opacity corrects part of the flux excess for wavelengths below $\sim 3000 \AA$, while giving quite a good estimate of the blanketed continuum distribution for longer wavelengths. On average, the correction procedure gives fluxes within $10 \%$ down to $\sim 4000 \AA$, or even below $1 \%$ in the wavelength range of the lines under consideration; the discrepancy with NextGen fluxes rises above a factor two at $\lambda<3000 \AA$. The likely cause of this residual difference lies in the strong variation of the $\kappa_{\mathrm{NLB}}$ at those wavelengths, which makes Eq. (2) less accurate.

Using the same approach for the whole grid of models we find that the continuum flux calculated adopting the total opacity of Eq. (3) agrees quite well with the corresponding NextGen flux for all the models, in the same range of wavelengths. Furthermore, we tested that, imposing HSE and using the resulting electron densities in the atmospheric models, the method gives essentially the same results.

Even if the method described above is quite general and can be applied to any atmospheric structure, it has the disadvantage of being model-dependent (that is, the $f_{\text {corr }}(\lambda)$ function has to be calculated for each model as a discrete function of frequency by computing the $\left.\ln \left(F_{\mathrm{NLB}} / F_{\mathrm{LB}}\right)\right)$.

In order to find a smoothed opacity-function, we fitted the measured $\ln \left(F_{\mathrm{NLB}} / F_{\mathrm{LB}}\right)$ functions with Gaussian curves:

$y(\lambda)=A \mathrm{e}^{\frac{-(\lambda-B)^{2}}{C}}+D$.

The flux distribution obtained using the Gaussian fit instead of the discrete $f_{\text {corr }}$ opacity function is shown in Fig. 2 (dot-dashed line). As we can see, the Gaussian fit can be considered a good approximation of the discrete $f_{\text {corr }}$ function, giving very similar UV excess corrections.

By fitting the logarithm of the parameters $A, B, C$ and $D$ of Eq. (4), obtained for the whole grid of atmospheric models, with a linear function of $\log T_{\text {eff }}, \log g$, and $[\mathrm{A} / \mathrm{H}]$, we obtain:

$\log A=-3.06 \log T_{\text {eff }}-0.0030 \log g+0.25[\mathrm{~A} / \mathrm{H}]+11.85$
$\log B=-1.08 \log T_{\text {eff }}-0.013 \log g+0.022[\mathrm{~A} / \mathrm{H}]+7.44$
$\log C=2.59 \log T_{\text {eff }}-0.020 \log g-0.0019[\mathrm{~A} / \mathrm{H}]-3.27$
$\log D=-0.10 \log T_{\text {eff }}-0.0060 \log g+0.011[\mathrm{~A} / \mathrm{H}]+0.24$

These equations, together with Eq. (4), allow us to obtain an estimate of the $f_{\text {corr }}$ function for a wide range atmospheric model.

\subsection{Effects on line formation}

In order to highlight the importance of a correct estimate of the UV continuum, we have considered the formation of the profiles of CaII H $(\lambda=3968 \AA)$, CaII $\lambda 8662$ (the redmost component of the IRT), Na I D $(\lambda=5896 \AA)$, K I (component at $\lambda=7699 \AA)$ and $\operatorname{Li}$ I $(\lambda=6708 \AA)$, using the original and the modified opacity of Eq. (3) (see Fig. 3).

It should be stressed once more how this approach is only appropriate for the analysis and fitting of highresolution spectra of strong lines, not for the construction of grids of stellar atmospheric models. The example of the Li I lines described above shows how the application of this method allows for the correction of UV excess in non-lineblanketed synthetic spectra, which could otherwise affect NLTE synthetic lines in the visible or IR range. It should also be noted that this method is flexible enough to allow the use of estimated line blanketing from observed UV spectra (e.g. IUE), rather than from theoretical spectral distribution, such as the NextGen spectra adopted as a reference in this paper.

The behaviour of the other components in the multiplets is essentially identical.

Note that most of these lines are known to be chromospherically and, in an active-star treatment, their analysis might require radiative transfer calculations in complete atmospheric models, going from photosphere up to corona. 

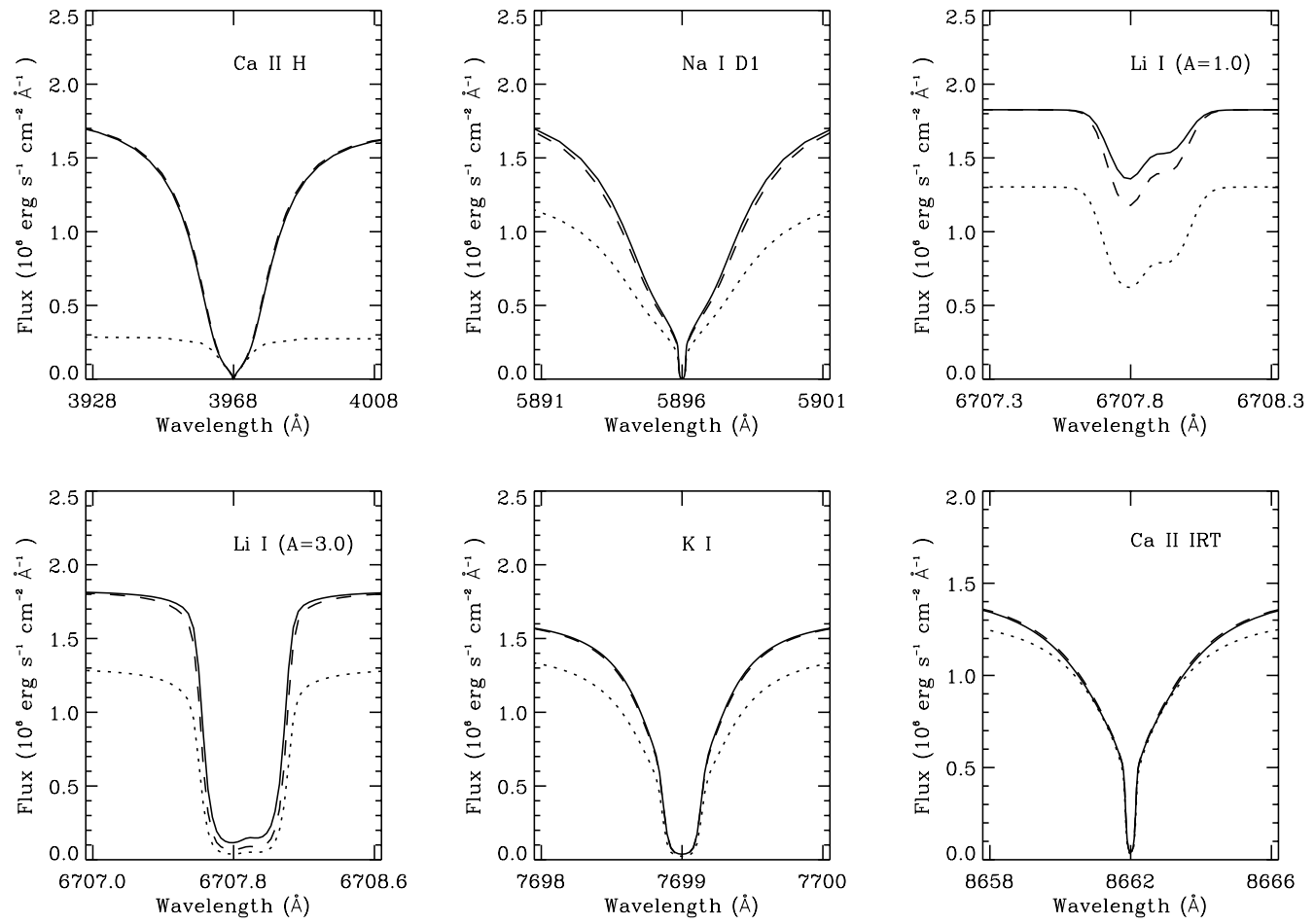

Fig. 3. The calculated Ca II H, Ca II IRT (component at $8662 \AA$ ), Na I D 1 , K I (component at $7699 \AA$ ) and Li I lines obtained without (solid line) and with (dotted line) the additional opacity for the $T_{\text {eff }}=4200 \mathrm{~K}, \log g=4.0$ and $[\mathrm{A} / \mathrm{H}]=0.0 \mathrm{model}$. Dashed line shows the profiles obtained by applying the opacity correction in the range $\lambda \leq 3500 \AA$ only.

Table 1. Equivalent Widths (in $\AA$ ) obtained with the original, the Eq. (3), and the Eq. (3) for $\lambda \leq 3500 \AA$ opacity distributions. In brackets we also give the rates $E W_{\mathrm{NLTE}} / E W_{\mathrm{LTE}}$.

\begin{tabular}{lrrr}
\hline & $E W_{\text {noadd }}$ & $E W_{3500}$ & $E W_{\text {add }}$ \\
Ca II H & $33.83(1.02)$ & $33.04(1.00)$ & $10.42(0.99)$ \\
Ca II $\lambda 8662$ & $3.21(1.03)$ & $3.12(1.00)$ & $2.68(1.00)$ \\
Na I D & $5.62(0.87)$ & $5.92(0.92)$ & $5.97(1.15)$ \\
K I $\lambda 7699$ & $0.77(1.00)$ & $0.79(1.03)$ & $0.88(1.30)$ \\
$\operatorname{Li}$ I $\left(A_{\mathrm{Li}}=1\right)$ & $0.062(0.34)$ & $0.090(0.49)$ & $0.15(0.99)$ \\
$\operatorname{Li~I}\left(A_{\mathrm{Li}}=3\right)$ & $0.47(0.88)$ & $0.52(0.97)$ & $0.57(1.20)$
\end{tabular}

Therefore, our calculations, in which we make use of photospheric models, can be applied to non-active stars only; for active stars a similarly detailed analysis is required.

Figure 3 shows how the line profiles (in absolute flux) change drastically when we consider the corrected opacity in the NLTE radiative transfer calculations. It is worthwhile noticing that, except for the Li I line, all the line profiles are mainly affected in the wings, while the line cores remain unchanged by the opacity modification.

This indicates that the changes in line profiles are due to an enhancement of the opacity in the wavelength range of the line, which has the effect of depressing the local "continuum" level. In agreement with this interpretation, we can see that the line profiles obtained by applying the opacity correction in the $\lambda \leq 3500 \AA$ range only (dashed line in Fig. 3) remain practically unchanged with respect to the original profiles (solid line). Obviously, this local effect cannot be neglected when normalising to the local "continuum" level, for instance when calculating equivalent widths (as in Table 1).

As far as the Li I line is concerned, our calculations indicate that the profile is affected both by the local change of the opacity but also by the NLTE effect of the UV radiation field which produce changes in the line core, especially in low abundance atmospheres (see Fig. 3).

The method described above will be applied in future analyses in order to correct the continuum UV excess which could affect the synthetic NLTE lines, as seen in the previous section.

Acknowledgements. We wish to thank Prof. G. Severino and Dr. A. C. Lanzafame for their critical reading of the manuscript. We thank the anonymous referee for useful comments and suggestions. This work was funded by MURST, CoFin 1998/2000 whose financial supports are gratefully acknowledged. We thanks M. Capaccioli for financial support from OAC.

\section{References}

Allard, F., \& Hauschildt, P. H. 1995, ApJ, 445, 433

Anderson, L. S. 1989, ApJ, 339, 588

Andretta, V., Doyle, J. C., \& Byrne, P. B. 1997, A\&A, 322, 266

Barman, T. S., Hauschildt, P. H., Short, C. I., \& Baron, E. 2000, ApJ, 537, 946 
Carlsson, M. 1986, A Computer Program for Solving MultiLevel Non-LTE Radiative Transfer Problems in Moving or Static Atmospheres, Report No. 33, Uppsala Astronomical Observatory

Carlsson, M., Rutten, R., Bruls, J., \& Shchunkina, N. 1994, A\&A, 288, 860

Chmielewski, Y. 2000, A\&A, 353, 666

Drake, J. 1991, MNRAS, 251, 369

Falchi, A., \& Mauas, P. 1998, A\&A, 336, 281

Grevesse, N., \& Anders, E. 1991, in Solar Interior and Atmosphere, ed. A. Cox, W. Livingston, \& M. Matthews, 1227

Hauschildt, P. H., Allard, F., Ferguson, J., Baron, E., \& Alexander, D. 1999, ApJ, 525, 871
Hubeny, I., \& Lanz, T. 1995, ApJ, 439, 875

Kurucz, R. L. 1992, in IAU Symp. 149, The Stellar Population of Galaxies, ed. B. Barbuy, \& A. Renzini (Kluwer), 225

Mauas, P., Falchi, A., Pasquini, L., \& Pallavicini, R. 1997, A\&A, 326, 249

Prieto, C. A., \& Lambert, D. L. 2000, A\&A, in press

Schweitzer, A., Hauschildt, P. H., \& Baron, E. 2000, ApJ, 541, 1004

Short, C. I., \& Doyle, J. C. 1997, A\&A, 326, 287

Smith, G., \& Drake, J. 1990, A\&A, 231, 125

Thomas, R. N. 1957, ApJ, 125, 260

Tripicchio, A., Gomez, M., Severino, G., et al. 1999, A\&A, 345, 915 